\title{
Site of Accessory Pathway Block After Radiofrequency Catheter Ablation in Patients with the Wolff-Parkinson-White Syndrome
}

\author{
HUGH CALKINS, M.D., CHING MANN, D.O., STEVEN KALBFLEISCH, M.D., \\ JONATHAN J. LANGBERG, M.D., and FRED MORADY, M.D. \\ From the Division of Cardiology, Department of Internal Medicine, \\ University of Michigan Medical Center, Ann Arbor, Michigan
}

\begin{abstract}
Site of Accessory Pathway Block. Introduction: Recent studies have demonstrated that the most common site of accessory pathway conduction block following the introduction of a premature atrial stimulus during atrial pacing is between the accessory pathway potential and the ventricular electrogram, consistent with block at the ventricular insertion of the accessory pathway. However, no prior study has evaluated the site of conduction block during radiofrequency catheter ablation procedures. Therefore, the objective of this study was to determine the site of conduction block after catheter ablation of accessory pathways by analyzing and comparing the local electrograms recorded before and after radiofrequency energy delivery at successful ablation sites.

Methods and Results: The electrograms evaluated in this study were obtained from 85 consecutive patients who underwent successful radiofrequency catheter ablation of a manifest accessory pathway. The $\mathbf{5 0}$ left free-wall accessory pathways were ablated using a ventricular approach and the 35 right free-wall or posteroseptal accessory pathways were ablated using an atrial approach. The characteristics of local electrograms recorded immediately before and immediately after successful ablation of the accessory pathway were determined in each patient. The site of accessory pathway block was determined by comparing the amplitude, timing, and morphology of the local electrograms at successful sites of radiofrequency catheter ablation before and after delivery of radiofrequency energy. A putative accessory pathway potential was present at the successful target site in $\mathbf{7 4}$ of the 85 patients $(87 \%)$. Conduction block occurred between the atrial electrogram and the accessory pathway potential in 66 patients $(78 \%)$ and between the accessory pathway potential and the ventricular electrogram in eight patients $(\mathbf{9 \%})$. The site of block could not be determined in 11 patients $(13 \%)$ in whom an accessory pathway potential was absent. Conduction block occurred most frequently between the atrial electrogram and the accessory pathway potential regardless of accessory pathway location. No electrogram parameter or accessory pathway characteristic was predictive of the site of conduction block.

Conclusion: The results of this study demonstrate that conduction block occurs most frequently between the local atrial electrogram and the accessory pathway potential during radiofrequency catheter ablation of accessory pathways. This is true regardless of whether the accessory pathway is ablated from the atrial or ventricular aspect of the mitral or tricuspid annulus. (J Cardiovasc Electrophysiol, Vol. 5, pp. 20-27, January 1994)
\end{abstract}

Wolff-Parkinson-White syndrome, radiofrequency energy, catheter ablation

Address for correspondence: Hugh Calkins, M.D., Carnegie Room 530, Johns Hopkins Hospital, 601 North Wolfe Street, Baltimore, MD 21210. Fax: 410-614-1345.

Manuscript received 30 April 1993; Accepted for publication 25 August 1993
Introduction

Recent studies have demonstrated that the most common site of accessory pathway conduction block following the introduction of a premature 
atrial stimulus during atrial pacing is between the accessory pathway potential and the ventricular electrogram, consistent with conduction block at the ventricular insertion of the accessory pathway. ${ }^{1,2}$ This observation suggests that the ventricular insertion of accessory pathways may be more susceptible to destruction than is the atrial insertion. However, no prior studies have characterized the site of conduction block during radiofrequency catheter ablation of accessory pathways.

Therefore, the objective of this study was to determine the site of conduction block during radiofrequency catheter ablation of accessory pathways by analyzing and comparing the local electrograms recorded before and after radiofrequency energy delivery at successful ablation sites.

\section{Methods}

\section{Patient Characteristics}

The electrograms evaluated in this study were obtained from 85 consecutive patients who underwent successful radiofrequency catheter ablation of a manifest accessory pathway at the University of Michigan Medical Center and in whom stable electrograms were recorded at the successful ablation site before and after radiofrequency energy delivery. Each patient had a single accessory pathway. There were 54 men and 31 women, and their mean age was $34 \pm 17$ years. Eighty-two patients had no evidence of structural heart disease, one had Ebstein's anomaly, one had a dilated cardiomyopathy, and one had coronary artery disease.

\section{Accessory Pathway Characteristics}

Each patient enrolled in this study had a single manifest accessory pathway. Fifty accessory pathways were located in the free wall of the left ventricle, 15 were posteroseptal, and 20 were located in the free wall of the right ventricle. The mean anterograde effective refractory period was $274 \pm$ $66 \mathrm{msec}$. The mean accessory pathway anterograde block cycle length was $293 \pm 77 \mathrm{msec}$ and the mean retrograde block cycle length was $287 \pm 57 \mathrm{msec}$.

\section{Electrophysiologic Testing and Catheter Ablation}

The techniques used for electrophysiologic testing and radiofrequency catheter ablation at the University of Michigan Medical Center have previously been published in detail. ${ }^{3,4}$ Following preliminary localization of the accessory pathway, precise mapping was performed with a 7 French catheter, which had a 4-mm distal electrode, 2-mm interelectrode spacing, and a deflectable tip (Mansfield Webster, Burlington, MA, USA, or EP Technologies, Inc., Mountain View, CA, USA). Left free-wall accessory pathways were approached from the ventricular aspect of the mitral annulus. Right-sided and posteroseptal accessory pathways were approached from the atrial side of the tricuspid annulus. Bipolar electrograms were recorded using the distal pair of electrodes of the ablation catheter. The electrograms were recorded during sinus rhythm, filtered at 50 to $500 \mathrm{~Hz}$, amplified at a gain of $20 \mathrm{~mm} / \mathrm{mV}$, and recorded at paper speeds of 100 or $200 \mathrm{~mm} / \mathrm{sec}$ using a SiemensElema (Solna, Sweden) Mingograph 7 recorder.

Target sites for ablation were identified by the presence of discrete atrial and ventricular electrograms, early ventricular activation relative to onset of the delta wave, and/or a discrete electrogram suggestive of an accessory pathway potential preceding the QRS complex.

\section{Electrogram Analysis}

The characteristics of local electrograms recorded immediately before and immediately after successful ablation of the accessory pathway were determined in each patient. The position of the tip of the ablation catheter was continuously monitored with fluoroscopic imaging during delivery of radiofrequency energy. Electrograms from those patients in whom catheter dislodgment was observed were excluded from analysis. Each electrogram was analyzed independently in blinded fashion by two of the authors. If their analysis differed significantly, the electrogram was analyzed by a third author and a consensus was reached.

The amplitude, morphology, and timing of the atrial, accessory pathway, and ventricular components of each local electrogram were determined. Morphological features that were evaluated included the presence of continuous electrical activity and the presence of an accessory pathway potential. An electrogram was classified as continuous if a $<5$ msec isoelectric segment was present between the atrial and ventricular components of the local electrogram. Determination of the presence of an accessory pathway potential was based strictly on morphological features of the local electrogram and were not verified with pacing maneuvers. ${ }^{1,2}$ Local electrograms were classified as demonstrating an 
accessory pathway potential if a discrete deflection was observed that preceded the onset of the QRS complex. The onset and activation time of the accessory pathway potential and atrial and ventricular components of each local electrogram were measured. The electrogram onset was defined as the first deflection from baseline with a slope $>45^{\circ}$ at a paper speed of $100 \mathrm{~mm} / \mathrm{sec}^{5}$ The activation time was defined as the point of maximal amplitude of the local electrogram. ${ }^{5,6}$ Once the onset and activation time of each component of the local electrogram were determined, the following intervals were measured: onset of the atrial electrogram to onset of the ventricular electrogram (AoVo interval); onset of an accessory pathway (K) potential to onset of the ventricular electrogram (KoVo inter$\mathrm{val}$ ); and the onset of the ventricular electrogram to onset of the QRS complex (VoQRS interval). These intervals were also determined based on activation times (AaVa, KaVa, and VaQRS intervals).

The site of conduction block was determined by comparing the amplitude, timing, and morphology of the local electrograms at successful ablation sites before and after delivery of radiofrequency energy. ${ }^{7}$ The site of conduction block was classified as between the accessory pathway potential and the local ventricular electrogram if the amplitude, timing, and morphology of the atrial and accessory pathway components of the local electrogram were unchanged after ablation of the accessory pathway (Fig. 1). A significant change in amplitude was defined as an increase or decrease in amplitude of one of the major components of the electrogram by more than $50 \%$. Conversely, the site of block was classified as between the local atrial electrogram and the accessory pathway potential if the presumed accessory pathway potential was no longer present after ablation (Fig. 2). The site of block was also classified as occurring between the local atrial electrogram and the accessory pathway potential if the amplitude of the presumed atrial electrogram decreased by more than $50 \%$, consistent with disappearance of an accessory pathway potential that had merged with the atrial electrogram (Fig. 3). The site of conduction block was classified as unknown if an accessory pathway potential was not observed before radiofrequency energy delivery and if the atrial electrogram remained unchanged after ablation of the accessory pathway.

\section{Statistical Analysis}

Values are expressed as mean $\pm 1 \mathrm{SD}$. The relationship between site of block and accessory path- way location was determined using Fisher's exact test with correction for multiple comparisons. Determination of the relationship between the AV ratio and the timing of the accessory pathway potential with the site of conduction block was performed using a nonpaired Student's $t$-test. Paired $t$-tests were used to compare the characteristics of electrograms before and after radiofrequency energy delivery. A P value $<0.05$ was considered significant.

\section{Results}

\section{Electrogram Characteristics at Successful Ablation Sites}

The characteristics of local electrograms recorded at successful ablation sites immediately before and after radiofrequency energy delivery are shown in Table 1. Accessory pathway potentials were observed at $85 \%$ of successful ablation sites before catheter ablation and at $9 \%$ of ablation sites after catheter ablation $(\mathrm{P}<0.001)$. Continuous electrical activity was present at $54 \%$ of successful ablation sites before catheter ablation and was uniformly absent after ablation $(\mathrm{P}<$ $0.001)$. All measured conduction intervals increased after ablation, consistent with elimination of conduction through the accessory pathway $(\mathrm{P}<0.001)$.

The electrogram amplitudes that were recorded at successful ablation sites are shown in Table 2. Neither the amplitudes of the atrial or ventricular electrograms nor the AV ratio changed significantly after delivery of radiofrequency energy.

\section{Site of Conduction Block}

Conduction block occurred between the atrial electrogram and the accessory pathway potential in 66 patients $(78 \%)$ and between the accessory pathway potential and the ventricular electrogram in 8 patients $(9 \%)$. The site of block could not be determined in 11 patients $(13 \%)$ in whom an accessory pathway potential was not present before ablation.

Conduction block occurred most frequently between the atrial electrogram and the accessory pathway potential at all accessory pathway locations (Table 3). However, conduction block between the accessory pathway potential and the ventricular electrogram was observed more frequently after ablation of posteroseptal accessory pathways than 


\section{Pre Ablation}

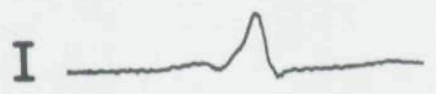

$\mathrm{ABL}$

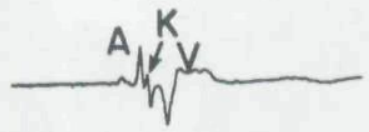

HBE

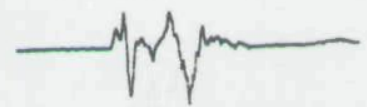

RVA



III

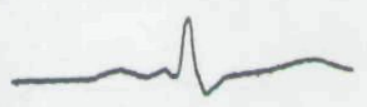

Post Ablation
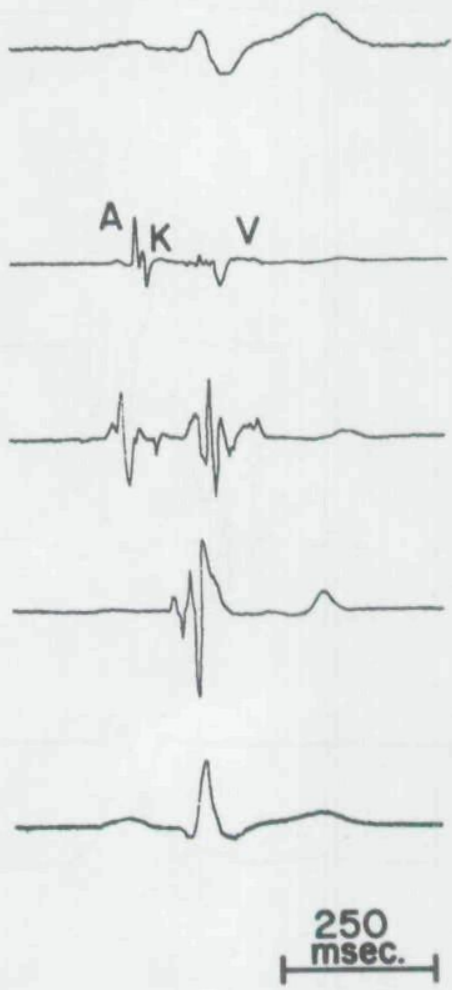

Figure 1. Recordings obtained at a successful ablation site immediately prior to (left panel) and following (right panel) radiofrequency energy delivery of a manifest right lateral accessory pathway. Shown are surface leads I and III, and the intracardiac electrograms recorded at the His-bundle position (HBE), right ventricular apex (RVA), and at the ablation site $(A B L)$ during sinus rhythm. Prior to ablation, distinct atrial, accessory pathway $(K)$, and ventricular electrograms are observed. Following ablation, the atrial electrogram and accessory pathway potential remain unchanged, consistent with conduction block between the accessory pathway potential and the ventricular electrogram.

after ablation of left free-wall accessory pathways $(\mathrm{P}=0.003)$.

The site of conduction block was not related to the timing of the accessory pathway potential, the relative amplitude of the atrial or ventricular electrogram at the ablation site, or to the electrophysiologic properties of the accessory pathway (Table 4). The mean anterograde effective refractory period of accessory pathways in which block occurred between the atrial electrogram and the accessory pathway potential was no different than in those in which block occurred between the accessory pathway potential and the ventricular electrogram or in those in which a site of block could not be determined due to the absence of an accessory pathway potential. Similarly, there was no relationship between the block cycle length of the accessory pathway and the site of block induced by ablation.

\section{Discussion}

\section{Main Findings}

The results of this study demonstrate that conduction block occurs most frequently between the local atrial electrogram and the accessory pathway potential during radiofrequency catheter ablation of accessory pathways. This observation suggests that catheter ablation does not result in selective destruction of the ventricular insertion of the accessory pathway.

\section{Identification of Accessory Pathway Potentials}

In this study, accessory pathway potentials were identified only based on morphology. Although pacing maneuvers have been described to verify the presence of an accessory pathway potential by 


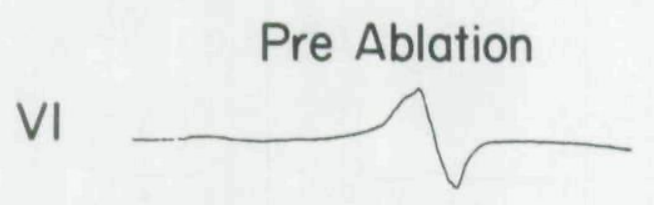

\section{Post Ablation}
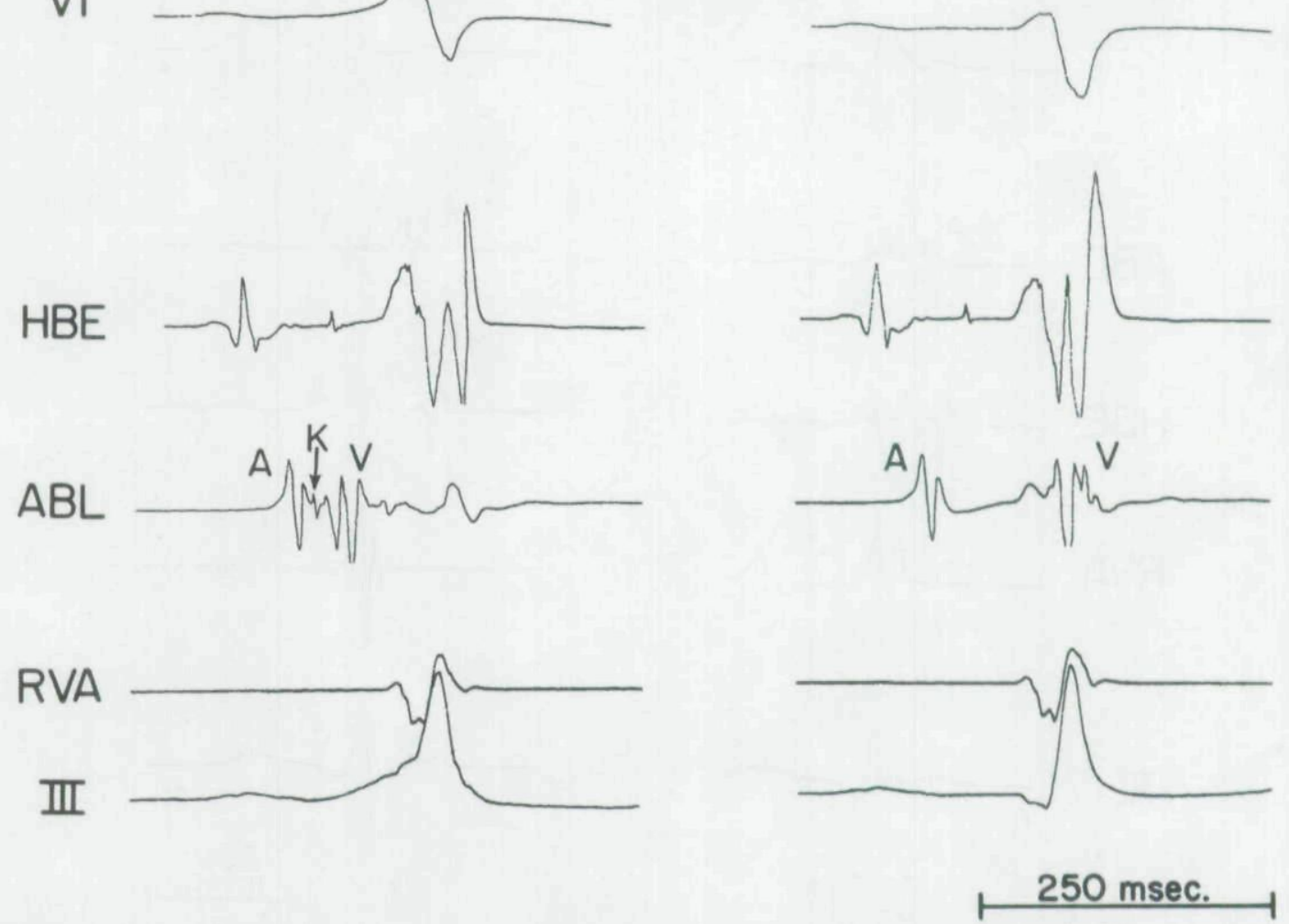

Figure 2. Recording obtained immediately prior to (left panel) and following (right panel) radiofrequency energy delivery at a successful ablation site of a manifest left anterolateral accessory pathway. Shown are surface leads VI and III, and the intracardiac electrograms recorded at the His-bundle position (HBE), right ventricular apex (RVA), and at the ablation site $(A B L)$ during sinus rhythm. Prior to ablation, distinct atrial, accessory pathway $(K)$, and ventricular electrograms are observed. Following ablation, the atrial electrogram remains unchanged and the $K$ potential is not present, consistent with conduction block between the atrial electrogram and the accessory pathway potential.

dissociating the suspected accessory pathway potential from both the atrial and ventricular components of the local electrogram,,$^{1,2}$ these techniques were not used in this study. The utility of identifying an accessory pathway potential based on morphology and timing was demonstrated in a previous study, ${ }^{8}$ which reported that accessory pathway potentials identified on this basis are independent predictors of success during radiofrequency catheter ablation. However, the techniques used to identify accessory pathway potentials in this study have not been validated and as such represent a limitation to this study.

\section{Determinants of the Site of Conduction Block}

In this study, left free-wall accessory pathways were ablated by positioning the ablation catheter across the aortic valve and against the ventricular aspect of the mitral annulus whereas right freewall and posteroseptal accessory pathways were ablated by positioning the ablation catheter on the atrial aspect of the tricuspid annulus or near the coronary sinus os. Although these two different approaches might be predicted to result in differing sites of conduction block, the findings of this study demonstrate that conduction block occurs most frequently between the local atrial electrogram and the accessory pathway potential, regardless of the site of radiofrequency energy delivery, and that no electrogram parameter or accessory pathway characteristic was predictive of the site of conduction block. This observation suggests that catheter ablation does not result in selective destruction of the ventricular insertion of the accessory pathway but is compatible with ablation of the 


\section{Pre Ablation}
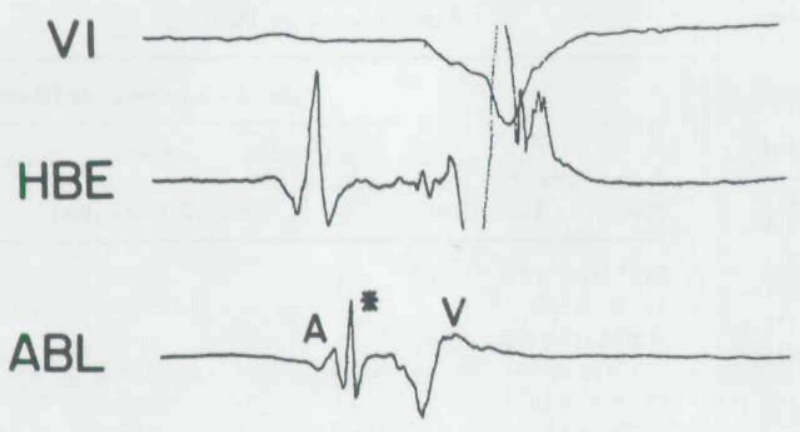

III

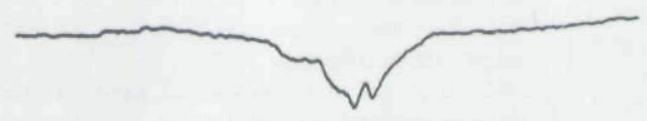

Post Ablation


$250 \mathrm{msec}$.

Figure 3. Recording obtained at a successful ablation site immediately prior to (left panel) and following (right panel) radiofrequency energy delivered to a manifest left posterior accessory pathway. Shown are surface leads VI and III, and the intracardiac electrograms recorded at the His-bundle position (HBE) and at the ablation site $(A B L)$ during sinus rhythm. Prior to ablation, a fractionated atrial electrogram is observed. Following ablation the atrial electrogram has decreased markedly in size and the large late component has disappeared, suggesting that the atrial electrogram and accessory pathway potential (asterisk) were fused. This pattern is consistent with conduction block between the atrial electrogram and the accessory pathway potential.

atrial insertion of the accessory pathway, the accessory pathway itself, both the accessory pathway and its atrial insertion, or both the accessory pathway and its ventricular insertion.

The apparent resistance of the ventricular insertion to radiofrequency ablation observed in this study is unlikely to be explained by the "impedance mismatch" hypothesis, which proposes that the relatively small amount of current that is conducted through the accessory pathway is unable to excite the ventricular myocardium because of the large muscle mass of the ventricle and the presence of low resistance intercellular connections. ${ }^{9,10}$ This hypothesis would predict that the ventricular insertion of the accessory pathway would be more vulnerable to anterograde conduction block than is the atrial insertion or the accessory pathway itself.

The resistance of the ventricular insertion to radiofrequency ablation may be explained by the anatomical observations that accessory pathways tend to arborize and course apically, away from the AV valve ring, before inserting into the ventricular myocardium. ${ }^{11-13}$ The arborization of the accessory pathway may result in inability of the relatively small lesion created with radiofrequency energy to ablate each accessory pathway fiber. Similarly, the trajectory of accessory pathways may result in the ventricular insertion of the accessory pathway being farther from the ablation catheter than is the atrial insertion, even when the ablation catheter is positioned on the ventricular side of the annulus. It is also possible that the resistance of the ventricular insertion of the accessory pathway may result from changes in fiber orientation at the atrial/accessory pathway junction,,${ }^{14}$ or may result from some unknown inherent property of the accessory pathway itself or of the intervening tissue.

Although conduction block occurs most frequently between the atrial electrogram and the accessory pathway potential at all accessory pathway locations, conduction block between the accessory pathway potential and the local ventricular electrogram occurs more frequently during ablation of posteroseptal accessory pathways than during ablation of left free-wall accessory pathways. This difference may be attributable to the more complex anatomy of the posteroseptal space. ${ }^{15}$ 


\section{TABLE 1}

Characteristics of Successful Ablation Sites in 85 Patients

\begin{tabular}{lccc}
\hline & $\begin{array}{c}\text { Before } \\
\text { Ablation } \\
\text { (No. of } \\
\text { Patients) }\end{array}$ & $\begin{array}{c}\text { After } \\
\text { Ablation } \\
\text { (No. of } \\
\text { Patients) }\end{array}$ & P Value \\
\hline K potential & $72(85 \%)$ & $8(9 \%)$ & $<0.001$ \\
CEA & $46(54 \%)$ & $0(0 \%)$ & $<0.001$ \\
AoVo (msec) & $47 \pm 17 *$ & $101 \pm 34$ & $<0.001$ \\
AaVa (msec) & $52 \pm 19$ & $120 \pm 46$ & $<0.001$ \\
VoQRS (msec) & $15 \pm 15$ & $-8 \pm 23$ & $<0.001$ \\
VaQRS (msec) & $-6 \pm 19$ & $-54 \pm 21$ & $<0.001$ \\
KoVo (msec) & $23 \pm 13$ & - & - \\
KaVo (msec) & $35 \pm 18$ & - & - \\
\hline
\end{tabular}

$\mathrm{AP}=$ accessory pathway; $\mathrm{CEA}=$ continuous electrical activity; $A o V_{0}=$ interval from onset of atrial electrogram to onset of ventricular electrogram; $\mathrm{AaVa}=$ interval from activation of atrial electrogram to activation of ventricular electrogram; VoQRS = interval from onset of ventricular electrogram to onset of the QRS complex; VaQRS = interval from activation of ventricular electrogram to onset of QRS complex; KoVo = interval from onset of the accessory pathway potential to onset of ventricular electrogram; $\mathrm{KaVo}=$ interval from activation of the accessory pathway potential to activation of ventricular electrogram.

*Mean \pm SD.

\section{Comparison with Prior Studies}

No prior study has evaluated the site of block during radiofrequency catheter ablation procedures.

\section{TABLE 2}

Electrogram Amplitudes at Successful Ablation Sites

\begin{tabular}{lccc}
\hline Variable & $\begin{array}{c}\text { Before } \\
\text { Ablation }\end{array}$ & $\begin{array}{c}\text { After } \\
\text { Ablation }\end{array}$ & P Value \\
\hline
\end{tabular}

Left Free Wall Accessory Pathway $(\mathbf{N}=\mathbf{8 0})$

$\begin{array}{lccc}\text { A ampl }(\mathrm{mV}) & 0.4 \pm 0.3 & 0.4 \pm 0.3 & 0.5 \\ \mathrm{~K} \text { ampl }(\mathrm{mV}) & 0.6 \pm 0.7 & - & - \\ \mathrm{V} \text { ampl }(\mathrm{mV}) & 2.1 \pm 1.3 & 2.1 \pm 1.2 & 0.4 \\ \mathrm{~A} / \mathrm{V} \text { ratio } & 0.3 \pm 0.3 & 0.2 \pm 0.2 & 0.3\end{array}$

Right Free Wall Accessory Pathway $(\mathrm{N}=\mathbf{2 0})$

$\begin{array}{lccc}\text { A ampl }(\mathrm{mV}) & 0.6 \pm 0.6 & 0.6 \pm 0.6 & 0.5 \\ \mathrm{~K} \text { ampl }(\mathrm{mV}) & 0.5 \pm 0.8 & - & - \\ \mathrm{V} \text { ampl }(\mathrm{mV}) & 1.3 \pm 1.2 & 1.4 \pm 1.3 & 0.3 \\ \text { A/V ratio } & 1.1 \pm 1.7 & 1.3 \pm 1.8 & 0.2\end{array}$

Posteroseptal Accessory Pathway $(\mathrm{N}=15)$

\begin{tabular}{llcl} 
A ampl $(\mathrm{mV})$ & $0.5 \pm 0.4$ & $0.7 \pm 1.1$ & 0.3 \\
$\mathrm{~K}$ ampl $(\mathrm{mV})$ & $0.6 \pm 0.8$ & - & - \\
$\mathrm{V}$ ampl $(\mathrm{mV})$ & $1.8 \pm 1.7$ & $1.7 \pm 1.5$ & 0.4 \\
$\mathrm{~A} / \mathrm{V}$ ratio & $0.7 \pm 1.0$ & $1.0 \pm 1.2$ & 0.2 \\
\hline
\end{tabular}

$\mathrm{A}=$ atrial; $\mathrm{K}=$ accessory pathway potential; $\mathrm{V}=$ ventricular; $\mathrm{A} / \mathrm{V}=$ ratio of the amplitude of the atrial and ventricular electrogram.

Values are expressed as mean \pm SD.
TABLE 3

Relationship Between Site of Conduction Block and Accessory Pathway

\begin{tabular}{llll}
\hline & \multicolumn{3}{c}{ Site of Conduction Block } \\
\cline { 2 - 4 } & \multicolumn{1}{c}{ AK } & \multicolumn{1}{c}{ KV } & Unknown \\
$\begin{array}{l}\text { Accessory } \\
\text { Pathway Location }\end{array}$ & \multicolumn{2}{c}{ (No. of Patients) } \\
\hline $\begin{array}{c}\text { Left free wall } \\
(\mathrm{N}=50)\end{array}$ \\
$\begin{array}{c}\text { Right free wall } \\
(\mathrm{N}=20)\end{array}$ & $40(80 \%)$ & $1(2 \%)$ & $9(18 \%)$ \\
$\begin{array}{c}\text { Posteroseptal } \\
(\mathrm{N}=15)\end{array}$ & $17(85 \%)$ & $2(10 \%)$ & $1(5 \%)$ \\
\hline
\end{tabular}

$\mathrm{AK}=$ conduction block between the atrial electrogram and the accessory pathway potential; $\mathrm{KV}=$ conduction block between the accessory pathway potential and the ventricular electrogram.

*Conduction block occurred more frequently between the accessory pathway potential and the ventricular electrogram during ablation of posteroseptal accessory pathways than during ablation of left free-wall accessory pathways.

However, the findings of our study are in contrast to those of Kuck et al., ${ }^{7}$ who demonstrated that accessory pathway block during atrial premature stimulation occurs at the ventricular insertion of left free-wall and posteroseptal accessory pathways. The pathophysiological basis for the differing sites of spontaneous versus catheter ablation-induced sites of conduction block remains uncertain. Furthermore, the inability to precisely determine whether the atrial insertion, accessory pathway itself, both the accessory pathway and the

\section{TABLE 4}

Relationship Between Site of Conduction Block, Electrophysiologic Properties of the Accessory Pathway, and the Relative Amplitude and Timing of the Local Electrogram

\begin{tabular}{lcccc}
\hline & \multicolumn{4}{c}{ Site of Conduction Block } \\
\cline { 2 - 5 } Parameter & $\begin{array}{c}\mathbf{A K} \\
(\mathbf{N}=\mathbf{6 6})\end{array}$ & $\begin{array}{c}\text { KV } \\
(\mathbf{N}=\mathbf{8})\end{array}$ & $\begin{array}{c}\text { Unknown } \\
(\mathbf{N}=11)\end{array}$ & $\begin{array}{c}\mathbf{P} \\
\text { Value }\end{array}$ \\
\hline $\begin{array}{c}\text { AK/AV } \\
\text { ratio }\end{array}$ & $0.38 \pm 0.23$ & $0.32 \pm 0.13$ & - & 0.5 \\
$\begin{array}{c}\text { AV ratio } \\
\text { AP ERP } \\
\text { (msec) }\end{array}$ & $0.49 \pm 0.96$ & $0.72 \pm 0.89$ & $0.81 \pm 1.16$ & 0.5 \\
$\begin{array}{c}\text { AP BCL } \\
\text { (msec) }\end{array}$ & $281 \pm 73$ & $263 \pm 21$ & $245 \pm 23$ & 0.2 \\
\hline
\end{tabular}

$\mathrm{AK} / \mathrm{AV}$ ratio $=$ ratio of interval from onset of the atrial electrogram to onset of the accessory pathway potential and the interval from onset of the atrial electrogram to onset of the ventricular electrogram; AV ratio = ratio of the amplitude of the atrial electrogram to the amplitude of the ventricular electrogram at the ablation site; $\mathrm{AP}$ ERP $=$ accessory pathway effective refractory period; $\mathrm{AP} \mathrm{BCL}=\mathrm{ac}-$ cessory pathway anterograde block cycle length. 
atrial insertion, or both the accessory pathway and the ventricular insertion is ablated prevents us from determining whether the atrial or ventricular insertion of the accessory pathway is the preferable site for delivery of radiofrequency energy.

\section{Study Limitations}

There are several limitations to this study. First, a multipolar coronary sinus catheter was not used to record accessory pathway potentials throughout the course of the accessory pathway. Therefore, the results of this study allow us to determine only if the site of conduction block occurred between the atrial electrogram and the accessory pathway potential or between the accessory pathway potential and the ventricular electrogram that were recorded at the ablation site. A second limitation is that none of the accessory pathways in this series were mid-septal or anteroseptal in location. Therefore, it is not known whether the results of this study apply to mid-septal or anteroseptal accessory pathways. A third limitation, as described above, is that the accessory pathway potentials were not validated with pacing techniques. Thus, this analysis examines the response of presumptive accessory pathway potentials to ablation.

\section{References}

1. Jackman WM, Friday KJ, Yeung-Lai-Wah JA, et al: New catheter technique for recording left free-wall accessory atrioventricular pathway activation. Identification of pathway fiber orientation. Circulation 1988;78:598-610.

2. Jackman WM, Friday KJ, Fitzgerald DM, et al: Localization of left free-wall and posteroseptal accessory atrioventricular pathways by direct recording of accessory pathway activation. PACE 1989;12:204-213.

3. Calkins H, Sousa J, El-Atassi R, et al: Diagnosis and cure of the Wolff-Parkinson-White syndrome or paroxysmal supraventricular tachycardias during a sin- gle electrophysiologic test. $N$ Engl J Med 1991;324:1612-1618.

4. Calkins H, Langberg J, Sousa J, et al: Radiofrequency catheter ablation of accessory atrioventricular connections in 250 patients: Abbreviated therapeutic approach to Wolff-Parkinson-White syndrome. Circulation 1992;85:1337-1346.

5. Paul T, Moak JP, Morris C, et al: Epicardial mapping: How to measure local activation? PACE 1990;13:285-292.

6. Gallagher JJ, Kasel JH, Cox JL, et al: Techniques of intraoperative electrophysiologic mapping. Am J Cardiol 1982;49:221-240.

7. Kuck KH, Friday KJ, Kunze KP, et al: Sites of conduction block in accessory atrioventricular pathways. Circulation 1990;82:407-417.

8. Calkins H, Kim YN, Schmaltz S, et al: Electrogram criteria for identification of appropriate target sites for radiofrequency catheter ablation of accessory atrioventricular connections. Circulation 1992;85:565-573.

9. de la Fuente D, Sasyniuk B, Moe GK: Conduction through a narrow isthmus in isolated atrial tissue: A model of the WPW syndrome. Circulation 1971;44:803-809.

10. Inoue H, Zipes DP: Conduction over an isthmus of atrial myocardium in vivo: A possible model of Wolff-Parkinson-White syndrome. Circulation 1987;76:637-647.

11. Becker AE, Anderson RH, Durrer D, et al: The anatomical substrates of Wolff-Parkinson-White syndrome: A clinicopathologic correlation in seven patients. Circulation 1978;57:870-879.

12. Becker AE, Anderson RH: The Wolff-ParkinsonWhite syndrome and its anatomical substrates. Anat Rec 1981;201:169-177.

13. Truex RC, Bishof JK, Hoffman EL: Accessory atrioventricular muscle bundles of the developing human heart. Anat Rec 1958;131:45-53.

14. Spach MS, Miller WT III, Geselowitz DB, et al: The discontinuous nature of propagation in normal canine cardiac muscle: Evidence of recurrent discontinuities of intracellular resistance that affect the membrane currents. Circ Res 1981;48:39-54.

15. Sealy WC, Gallagher JJ: The surgical approach to the septal area of the heart based on experiences with 45 patients with Kent bundles. Cardiovasc Surg 1980;79:542-551. 
This document is a scanned copy of a printed document. No warranty is given about the accuracy of the copy. Users should refer to the original published version of the material. 